\title{
Effect of Jingqian Zhitong Fang on Serum Sex Hormone Levels in Women with Primary Dysmenorrhea
}

\author{
Na Dai, ${ }^{1}$ Ling Fang, ${ }^{1}$ Yu-bo Li, ${ }^{1}$ Yu-ming Wang, ${ }^{1}$ Ju Yin, ${ }^{2}$ and Bao-chan Pu \\ ${ }^{1}$ Tianjin State Key Laboratory of Modern Chinese Medicine, School of Traditional Chinese Materia Medica, \\ Tianjin University of Traditional Chinese Medicine, No. 312 Anshan West Road, Tianjin 300193, China \\ ${ }^{2}$ Obstetrics and Gynecology Hospital in Tianjin, Tianjin 300193, China
}

Correspondence should be addressed to Ling Fang; 13602177326@139.com

Received 23 December 2013; Revised 8 March 2014; Accepted 25 March 2014; Published 16 April 2014

Academic Editor: Ling Yang

Copyright (C) $2014 \mathrm{Na}$ Dai et al. This is an open access article distributed under the Creative Commons Attribution License, which permits unrestricted use, distribution, and reproduction in any medium, provided the original work is properly cited.

\begin{abstract}
Primary dysmenorrhea is a common gynecological disease garnering increasing attention and research. To investigate the clinical therapeutic effects of Jingqian Zhitong Fang (JQF) and the differences in serum sex hormone levels during the treatment of primary dysmenorrhea, we selected 30 healthy volunteers and 60 individuals with primary dysmenorrhea. On the third day of the menstrual cycle, we used ELISA to determine the levels of serum prolactin (PRL), follicle-stimulating hormone (FSH), luteinizing hormone $(\mathrm{LH})$, testosterone (TEST), progesterone (PROG), and estradiol $\left(\mathrm{E}_{2}\right)$ compared with normal levels and levels in the JQF group, the Western medicine group receiving continuous treatment during the first and third menstrual cycles, and the group followed up after the drug was stopped. We observed that after JQF treatment, the levels of the following hormones changed significantly: PRL, LH, TEST, and $\mathrm{E}_{2}$ levels decreased significantly and the PROG level increased significantly after treatment. After treatment with Western medicine, the serum levels of FSH, LH, PROG, and $\mathrm{E}_{2}$ showed no significant change. We conclude that the long-term effect of JQF treatment was better than that of Western medicine. JQF treatment of primary dysmenorrhea is related to adjustment of PRL, LH, TEST, and E2 hormone levels in the human body.
\end{abstract}

\section{Introduction}

Primary dysmenorrhea (PD) is characterized by cramping pelvic pain at the onset of menses, lasting for 48-72 hours during the menstrual cycle, and is highly prevalent (approximately 50\%) among adolescent girls [1,2]. According to an epidemiological sampling survey conducted in 1980, primary dysmenorrhea is a common gynecological disease. In China, the dysmenorrhea incidence was $33.1 \%$, with primary dysmenorrhea patients accounting for $53.2 \%$ and dysmenorrhea patients heavily influenced by the work accounting for $13.55 \%$ [3]. Because of dysmenorrhea, six-hundred million work days are lost annually in the USA, leading to economic losses of up to 200 million dollars [4].

The hormonal characteristics of humans have been reported. In the menstrual cycle, prostaglandins are smooth muscle stimulants [5]. In the luteal phase, the endometrium produces more prostaglandins. The $\mathrm{PGE}_{2}$ content is higher than $\mathrm{PGF}_{2 \alpha}$ content, which is opposite to that during the menstrual cycle. Powell et al. found that the levels of menstrual $\mathrm{PGF}_{2 \alpha}$ and $\mathrm{PGE}_{2}$ were significantly higher in women with dysmenorrhea than in healthy women [6]. The serum levels of estradiol $\left(\mathrm{E}_{2}\right)$, luteinizing hormone $(\mathrm{LH})$, and follicle-stimulating hormone (FSH) play a similar role in women with dysmenorrhea [7]. The abnormal hormone levels caused by primary dysmenorrhea can cause a variety of diseases. Progesterone (PROG) is a major progestational steroid that can decrease the contractility of uterine smooth muscle. High progesterone levels can decrease the incidence of breast cancer. In contrast, they can cause tumor formation [8]. Estradiol is an estrogen whose catechol metabolites that bind with proteins and nucleic acids can lead to cell damage and induce cancer [9]. Some studies have reported that primary dysmenorrhea may be caused by endometriosis, which can aggravate dysmenorrhea [10]. Patients with both adenomyosis and endometriosis show high levels of $\mathrm{E}_{2}$, FSH, and LH [7]. Therefore, it is necessary to study the relationship between the occurrence of dysmenorrhea and the disequilibrium of sex hormones. 
The Chinese herbal formula Jingqian Zhitong Fang (JQF) is considered to be an effective prescription for the treatment of PD. JQF originally came from Foshou san, which, in clinical practice, has been proven to treat cold retention and blood in gynecological diseases such as primary dysmenorrhea. Angelica is the main drug in JQF and could regulate menstrual pain. Angelica oil (including 70\% ligustilide), regardless of whether the uterus is normal and nonpregnant or has been treated with oxytocin, can relieve uterine smooth muscle pain [11]. Motherwort can regulate menstruation [12]. Chuanxiong was used in the clinical treatment of irregular menstruation, amenorrhea dysmenorrhea, and headache. And its main components: ferulic acid, tetramethylpyrazine, and ligustilide have an antispasmodic effect on smooth muscles and can lead to uterine smooth muscle relaxation $[13,14]$. Radix paeoniae Rubra is rich in glycosides, which can heat and cool blood, heal bruises, and relieve pain [15]. On the basis of experimental pharmacological studies, JQF can significantly inhibit oxytocin-induced cramping reflexes in mice and can also increase pain thresholds [16].

In this study, we collected plasma samples from the PD patients and volunteers and used ELISA to compare the different levels of six serum sex hormones. At the same time, we found variations in the changes in every sex hormone during treatment. After treatment, we compared the sex hormone levels between PD patients (JQF treatment group and Western medicine group) and healthy volunteers to determine whether the differences were important indicators of the clinical efficacy of JQF.

\section{Materials and Methods}

2.1. Study Population and Design. The protocol for this study was reviewed and approved by Tianjin University of Traditional Chinese Medicine. From January 2010 to December 2013, 30 volunteers without dysmenorrhea (age, 16-28 years; mean, $21.66 \pm 3.03$ years) and with body weight of $42-$ $69 \mathrm{~kg}$ (mean, $56.81 \pm 8.62 \mathrm{~kg}$ ) were selected. Sixty outpatients with primary dysmenorrhea were identified in the Obstetrics and Gynecology Hospital in Tianjin and Affiliated HealthCare Hospital of Tianjin University of Traditional Chinese Medicine and were randomly divided into the JQF treatment group and the Western medicine group. Those in the JQF group were aged $15-32$ years old (mean, $21.33 \pm 4.27$ years) with the course of disease lasting from 1 to 14 years (average, $6.60 \pm 3.81$ years) and mean body weight of $58.69 \pm 8.00 \mathrm{~kg}$. Those in the Western medicine group were aged 14 to 30 years (mean, $21.42 \pm 5.32$ years) with mean body weight of $58.48 \pm 9.25 \mathrm{~kg}$ and a course of disease lasting from 1.5 to 15 years (average, $6.64 \pm 3.70$ ). All primary dysmenorrhea patients were nonpregnant and showed no abnormalities upon gynecological examination. In the corpus luteum atrophy period, continuous treatment was administered during three menstrual cycles, with curative effects evaluated for the first menstrual cycle and the third menstrual cycle, and follow-up. At all time points, menstrual blood was collected on the third day of the menstrual cycle for detection of the levels of six serum sex hormones.
2.2. Drug Administration. JQF includes, among other components, Angelica sinensis, Rhizoma chuanxiong, and Radix paeoniae Rubra (confirmed by the Tianjin University of Traditional Chinese Medicine Plants Laboratory). To avoid possible biases resulting from differences in herbs produced in different areas, all necessary herbs were purchased together. The steps in JQF medication preparation are as follows: the herbs are mixed together according to their prescribed dosages, and water 10 times the volume of the herbs is added, with the mixture being boiled for $1 \mathrm{~h}$. The first extract is poured, the same volume of water is added, and the mixture is boiled for another hour. The second extract is poured and the two extracts are mixed. Finally, after concentration and drying, a solid extract is obtained. The ratio of solid extract to the herbs' dry weight was $24.6 \%$. Quantitative analysis of the chemical composition of JQF has been performed (see Supplementary Text 1 in Supplementary Material available online at http://dx.doi.org/10.1155/2014/876431). The primary dysmenorrhea group received doses of JQF or oral ibuprofen during the corpus luteum atrophy period (3-5 days before menstruation) with continuous treatment during three menstrual cycles. Dosage was discontinued in the fourth cycle.

2.3. Sample Preparation Detection Method. Blood samples were taken from volunteers on the third day of the menstrual cycle, placed in a water bath at $30^{\circ} \mathrm{C}$ for $30 \mathrm{~min}$ then centrifuged at $3600 \mathrm{~g}$ for $10 \mathrm{~min}$. The supernatant was injected into the ELISA kit and used for the quantitative determination of six serum sex hormone levels (PRL, FSH, $\mathrm{LH}$, TEST, PROG, and $\mathrm{E}_{2}$ ) on the third day of the menstrual cycle. According to the hypothalamic-pituitary-ovarian axis changes in the menstrual cycle, in the first to seventh days of menstruation, the levels of PRL, FSH, LH, PROG, and $\mathrm{E}_{2}$ showed little fluctuation, which was easy to manipulate, so venous blood was collected on the third day of the menstrual cycle.

2.4. Efficacy Criteria. According to the Guiding Principles of Clinical Research on Traditional Chinese Medicine for new drugs, issued by the Ministry of Health, People's Republic of China, we established the following dysmenorrhea scoring criteria: mild, dysmenorrhea symptom score of $<8$ points; moderate, dysmenorrhea symptom score from 8 to 13.5 points; and severe, dysmenorrhea symptom score of $>14$ points.

The posttreatment efficacy criteria were as follows: the dysmenorrhea symptoms disappeared or were significantly decreased, and comparison with pretreatment of dysmenorrhea symptoms showed a marked reduction of $>50 \%$ in the symptom score; the symptoms of dysmenorrhea were alleviated, and the dysmenorrhea symptom score was effectively decreased by $25 \%-50 \%$; or there was no obvious improvement in dysmenorrhea and other symptoms, with the dysmenorrhea symptom score decreased by $<25 \%$, thus rendering treatment ineffective.

2.5. Statistical Analyses. The measurement data were expressed as means \pm standard deviation $\left(\chi^{2} \pm S\right)$, with analysis 
TABLE 1: Comparison of women with primary dysmenorrhea in the first and third menstrual cycles with JQF and Western medicine treatment and follow-up after treatment $[n(\%)]$.

\begin{tabular}{lccccc}
\hline & $n$ & $\begin{array}{c}\text { Markedly } \\
\text { effective }\end{array}$ & Effective & Ineffective & $\begin{array}{c}\text { Total } \\
\text { efficiency }\end{array}$ \\
\hline J one cycle & 30 & $16(53.3)$ & $10(33.3)$ & $4(13.3)$ & 88.7 \\
J three cycles & 19 & $13(68.4)$ & $5(26.3)$ & $1(5.3)$ & 94.7 \\
J follow-up & 17 & $13(76.5)$ & $3(17.6)$ & $1(5.9)$ & 94.1 \\
W one cycle & 30 & $24(80.0)$ & $4(13.3)$ & $2(6.7)$ & 93.3 \\
W three & 20 & $14(70.0)$ & $4(20.0)$ & $2(10.0)$ & 90.0 \\
cycles & & $0(0.0)$ & $4(20.0)$ & $16(80.0)$ & 20.0 \\
W follow-up & 20 & $0(3)$
\end{tabular}

"W" stands for the Western medicine treatment group; "J" stands for the JQF treatment group.

of variance and correlation tests. The $\chi^{2}$ test was used for counting data, with $\alpha=0.05$, and data were analyzed with SPSS11.5 software. The correlation tests were analyzed with SIMCA 11.5 software.

\section{Results}

The results after JQF treatment for 1 month, 3 months, and follow-up showed that the total clinically effective rates were $88.7 \%, 94.7 \%$, and $94.1 \%$, respectively (Table 1 ). The dysmenorrhea symptom score decreased after treatment. Effects were weak after treatment for the "one menstrual cycle" group $(P<0.05)$, but there were significant effects of treatment for the "three menstrual cycles" and follow-up groups $(P<0.01)$ (Table 2). Venous blood was collected on the third day of the menstrual cycle, and the six serum sex hormone levels were measured. The results showed that the PRL, TEST, and $\mathrm{E}_{2}$ levels were higher in the dysmenorrhea group than in the healthy groups $(P<0.01)$. After treatment for one or three menstrual cycles, the three serum sex hormone levels were lower than those in the "no treatment" group $(P<0.01)$ and were almost the same as those in the healthy group. The FSH levels did not differ among all the groups (dysmenorrhea model group, treatment group, follow-up group, and the healthy group) $(P>0.05)$. Compared with the normal group, the LH level in the dysmenorrhea group increased $(P<0.05)$. There was no significant difference $(P>0.05)$ between the "one menstrual cycle" and dysmenorrhea groups, but the LH level decreased significantly in the "three menstrual cycles" and follow-up groups $(P<0.05)$. Compared with the normal group, the PROG level showed a downward trend in the dysmenorrhea group; however, after treatment, it expressed an upward trend in all the treatment groups (Table 3).

The total clinically effective rates at 1 month, 3 months, and follow-up after Western medicine treatment were $93.3 \%$, $90.0 \%$, and $20.0 \%$, respectively. (Table 1 ) The dysmenorrhea symptom scores were significantly decreased in the "one menstrual cycle" and "three menstrual cycles" groups $(P<$ 0.01 ); however, there was no difference between the pretreatment group and the follow-up group (Table 2). Compared with those in normal volunteers, the PRL, LH, TEST, and $\mathrm{E}_{2}$ levels were higher in the dysmenorrhea patients before
TABLE 2: The integral contrasts in primary dysmenorrhea after treatment.

\begin{tabular}{lcc}
\hline & JQF group $(n)$ & Western medicine group $(n)$ \\
\hline Before treatment & $11.52 \pm 3.36(30)$ & $11.86 \pm 2.98(30)$ \\
The first cycle & $7.35 \pm 3.15^{*}(30)$ & $8.86 \pm 2.76^{* *}(30)$ \\
The third cycle & $1.86 \pm 1.69^{* *}(19)$ & $2.56 \pm 1.54^{* *}(20)$ \\
Follow-up & $1.43 \pm 1.12^{* *}(17)$ & $11.88 \pm 4.13(20)$ \\
\hline
\end{tabular}

** Integral values of the degree of dysmenorrhea before and after treatment, $P<0.01 .{ }^{*} P<0.05$.

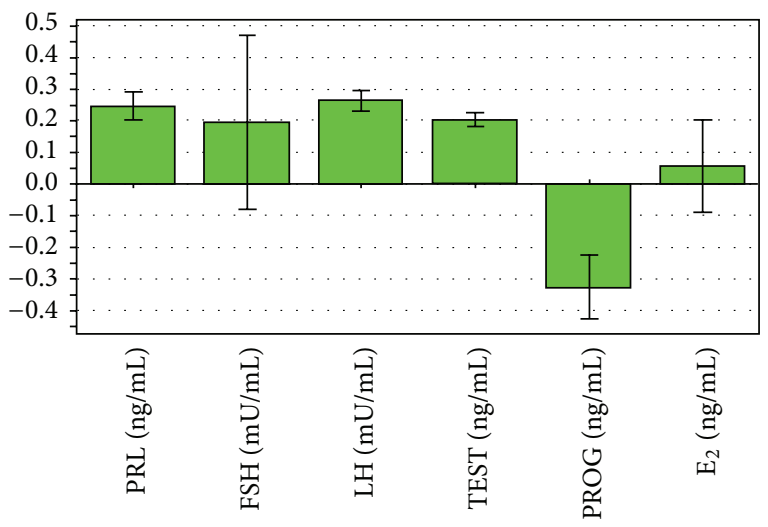

FIgURE 1: The correlation coefficients between the levels of six hormones and the scores for the extent of the disease (the bars indicate the confidence intervals of the coefficients. The coefficient is significant when the confidence interval does not cross zero.).

treatment $(P<0.01)$. After Western medicine treatment for the first and third menstrual cycles, the four hormone levels were decreased $(P<0.05)$. However, during follow-up, we found that $\mathrm{LH}$ and $\mathrm{E}_{2}$ had returned to pretreatment levels. There was no difference in FSH and PROG in the different groups. Western medicine treatment had a rapid onset of features, but the results from the follow-up showed that its long-term prognosis was poor.

The levels of six serum hormones in a certain time period have the combined effect of the degree of dysmenorrhea integration. Therefore, by correlation analysis with six kinds of hormone levels and dysmenorrhea integrals, we have established a regression equation: $Y=1.15125$ $+0.24804 X_{1}+0.19443 X_{2}+0.26509 X_{3}+0.20469 X_{4}-$ $0.32597 X_{5}+0.05783 X_{6}\left(Y\right.$, dysmenorrhea inregrals; $X_{1}$, PRL; $X_{2}$, FSH; $X_{3}, \mathrm{LH} ; X_{4}$, TEST; $X_{5}$, PROG; $\left.X_{6}, \mathrm{E}_{2}\right)$. PRL, FSH, LH, TEST, and $E_{2}$ positively associated with dysmenorrhea integrals and PROG negatively correlated with dysmenorrhea integral. The PRL, PROG, LH, and TEST have a larger impact of dysmenorrhea integral than $\mathrm{E}_{2}$ and $\mathrm{FSH}$ (Figure 1).

\section{Discussion}

JQF includes, among other components, Angelica sinensis, Rhizoma chuanxiong, and Radix paeoniae Rubra. Angelicae sinensis can enrich, supplement, and nourish blood. Rhizoma chuanxiong is regarded as a component that can result in improvements in the respiratory and cardiovascular systems. 


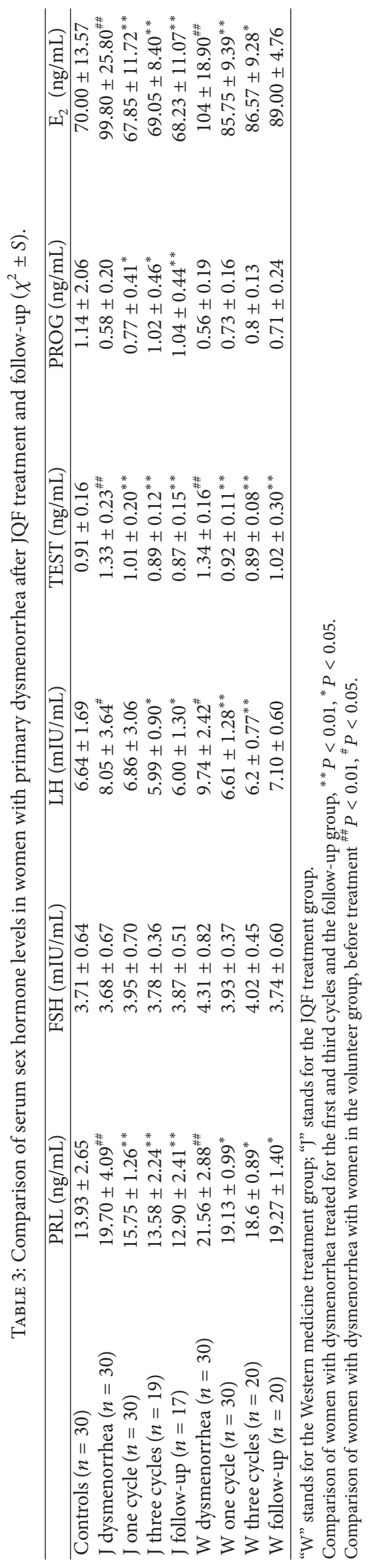




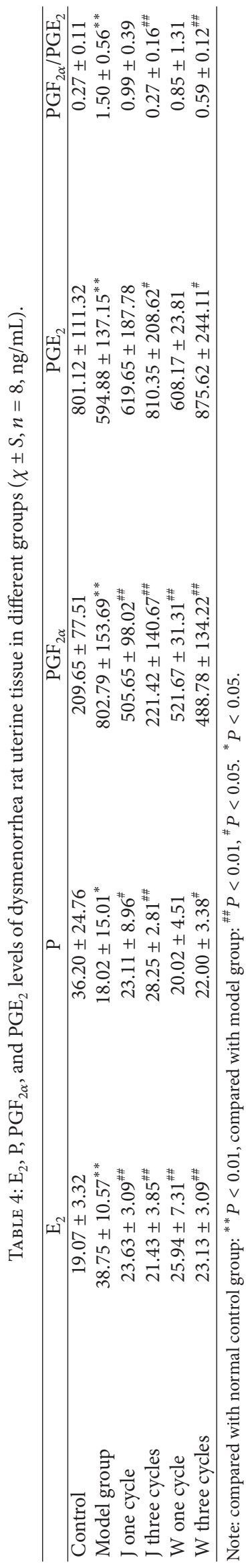


The complete prescription prevents diarrhea and results in improvement of the cardiovascular and respiratory systems.

Ibuprofen has analgesic, antipyretic, and anti-inflammatory effects and is one of a group of nonsteroidal antiinflammatory analgesics. Because it has a certain stimulus in the gastrointestinal tract, it can cause relative reduction of the side-effects of sustained-release formulations and can allow for the gradual release of drugs in the body. Ibuprofen sustained-release capsules are a prostaglandin synthetase inhibitor and can decrease the production of prostaglandins to prevent uterine contractions and spasms, thereby decreasing or eliminating dysmenorrhea. Their effects are rapid, and adverse reactions are mild. However, there are disadvantages in that symptoms can easily recur after treatment is discontinued.

The results of this study showed that, before and after treatment, the total efficiency and dysmenorrhea symptom scores of the JQF therapy group and Western medicine group showed significant differences in one cycle and three cycles. At follow-up, the patients were significantly better after JQF treatment than after Western medicine treatment, and there was a significant difference between the two groups.

Analysis of biochemical data showed that the serum endocrine index was significantly changed $(P<0.05)$ after JQF treatment. The PRL, LH, TEST, and $\mathrm{E}_{2}$ levels were significantly decreased, whereas the PROG levels were significantly increased after treatment. These data are consistent with our previous explore on trends of JQF treatment in dysmenorrhea animal models on the levels of $\mathrm{E}_{2}$ and $\mathrm{P}$. Further validated, JQF had a good therapeutic effect on primary dysmenorrhea (Table 4). It also showed an overall efficiency in regulating the biochemical parameters related to endocrinology [17, 18]. On JQF group, 11 patients were excluded in the third menstrual cycle, because they refused to take the medications or suffer from diseases. Sixteen patients were treated effectively. The integral in the fourth menstrual cycle was $1.43 \pm 1.12$ in the follow-up period. Compared with the first menstrual cycle $(7.35 \pm 3.15)$, the integral of the symptoms of dysmenorrhea was significantly decreased.

The luteal estrogen levels were significantly higher in the test group than in the control group, suggesting that the abnormal synthesis of endometrial PGs may be related to excessive estrogen [19]. The increased $\mathrm{E}_{2}$ level may have indirectly promoted the synthesis and release of $\mathrm{PGF}_{2 \alpha}$, resulting in uterine contractions and uterine ischemia or angiospasm, causing dysmenorrhea (Table 4). The endometrium can also produce estrogen, and high estrogen levels promote excessive uterine contractions and cause diseases [20]. Our study shows that the level of estrogen is positively correlated with dysmenorrhea. PROG is antagonistic to $\mathrm{E}_{2}$, meaning that the increasing content of PROG inhibits the production of $\mathrm{PGF}_{2 \alpha}$ to relieve uterine smooth muscle spasms. Such antagonism can also trigger dysmenorrhea indirectly via affecting the synthesis and release of other hormones, such as oxytocin, vasopressin, and endogenous opioid peptides [21].

\section{Conclusions}

In conclusion, estrogen can promote the pituitary to secrete PRL and inhibit the secretion of dopamine. Therefore, we can decrease DA levels to relieve the inhibition of PRL, whereas estrogen levels are high. Androgens, especially testosterone, can promote PRL secretion. LH subunits can cause increased PRL secretion. In the menstrual cycle, the secretion of PRL showed no significant change. Peripheral blood with a concentration of PRL is one of the contributors to menstrual disorders.

The results showed that changes in PRL, LH, TEST, PROG, and $\mathrm{E}_{2}$ concentrations are correlated with dysmenorrhea.

\section{Conflict of Interests}

The authors declare that there is no conflict of interests regarding the publication of this paper.

\section{Author's Contribution}

$\mathrm{Na}$ Dai and Ling Fang contributed equally to the work as cofirst authors.

\section{Acknowledgment}

This project was supported by The Research Programs of Application of Basic and Frontier Technology in Tianjin (13JCYBJC23900) and Tianjin University Undergraduates Teaching Quality and Teaching Reform Project (B07-1008).

\section{References}

[1] L. French, "Dysmenorrhea," American Family Physician, vol. 71, no. 2, pp. 285-291, 2005.

[2] A. R. Davis and C. L. Westhoff, "Primary dysmenorrhea in adolescent girls and treatment with oral contraceptives," Journal of Pediatric and Adolescent Gynecology, vol. 14, no. 1, pp. 3-8, 2001.

[3] The World of Traditional Medicine, Gynecology, Sciences Press, Beijing, China, 1999.

[4] M. Y. Dawood, "Nonsteroidal anti-inflammatory drugs and changing attitudes toward dysmenorrhea," The American Journal of Medicine, vol. 84, no. 5, pp. 23-29, 1988.

[5] V. R. Pickles, W. J. Hall, F. A. Best, and G. N. Smith, "Prostaglandins in endometrinm and menstrual fluid form normal and dysmenorrhoeic subjects," The Journal of Obstetrics and Gynaecology, vol. 72, pp. 185-192, 1965.

[6] A. M. Powell, W. Y. Chan, P. Alvin, and I. F. Litt, "Menstrual$\operatorname{PGF}(2 \alpha)$, PGE2 and TXA2 in normal and dysmenorrheic women and their temporal relationship to dysmenorrhea," Prostaglandins, vol. 29, no. 2, pp. 273-289, 1985.

[7] J.-L. Kang, X.-X. Wang, M.-L. Nie, and X.-H. Huang, "Efficacy of gonadotropin-releasing hormone agonist and an extendedinterval dosing regimen in the treatment of patients with adenomyosis and endometriosis," Gynecologic and Obstetric Investigation, vol. 69, no. 2, pp. 73-77, 2010.

[8] R. Kaaks, F. Berrino, T. Key et al., "Serum sex steroids in premenopausal women and breast cancer risk within the European Prospective Investigation into Cancer and Nutrition (EPIC)," Journal of the National Cancer Institute, vol. 97, no. 10, pp. 755$765,2005$.

[9] E. Pfeiffer, E. Graf, S. Gerstner, and M. Metzler, "Stimulation of estradiol glucuronidation: a protective mechanism against est- 
radiol-mediated carcinogenesis?" Molecular Nutrition and Food Research, vol. 50, no. 4-5, pp. 385-389, 2006.

[10] J. Hong, "The clinical observation of female pelvic blood flowing diagram to blood stasis dysmenorrhea and preliminary study of the application," Obstetrics and Gynecology, vol. 19, no. 4, article 203, 1984.

[11] R. Peng-fei and D. Yi, "Study progress on pharmacodynamics of DangGui and its active ingredients," Western Journal of Traditional Chinese Medicine, vol. 25, no. 9, pp. 125-128, 2012.

[12] G. Yujun and M. Aolei, "Analysis of the pharmacological effects of motherwort," Seek Medical and Ask the Medicine, vol. 10, no. 6, article 791, 2012.

[13] G. Jiansheng and G. Qianyao, "Advances in modern medicine pharmacology Chuanxiong," in Proceedings of the 5th National Symposium on Clinical Pharmacy, pp. 239-243, 2012.

[14] X. Xiuiqiong, Z. Ke, and Y. Rongli, "Progress of Chuanxiong volatile oil," Lishizhen Medicine and Materia Medica Research, vol. 18, no. 6, pp. 1508-1510, 2007.

[15] J. Lan-Xin, H. Hao, and L. Chang-Zhi, "Review in research of radix paeoniae rubra," Drug Evaluation Research, vol. 33, no. 6, pp. 233-236, 2010.

[16] L. Fang, X. Zhu, and C. Zhu, "The study of Mechanism of the three analgesia prescriptions previous to menstrual cycle to treat primary dysmenorrhrea," Chinese Herbal Medicine, vol. 41, no. 4, pp. 615-617, 2010.

[17] Y. Jin and Y. Hong, "Advancements in the chemical constituents and pharmacological effects of chuanxiong," Pharmacy and Clinics of Chinese Materia Medica, vol. 4, no. 3, pp. 44-48, 2013.

[18] K. Fang, X. Zhu, and J. Yin, "The study of mechanism of the three analgesia prescriptions previous to menstrual cycle to treat experimental rat model of dysmenorrhrea," Tianjin Traditional Chinese Medicine, vol. 27, no. 1, pp. 46-49, 2010.

[19] D. Huang, "The observation and summarization of TCM cycle therapy to treat primary dysmenorrhrea with 285 patients," Chinese Medicine Research, no. 6, pp. 17-18, 1995.

[20] T. Yamamoto, T. Noguchi, T. Tamura, J. Kitawaki, and H. Okada, "Evidence for estrogen synthesis in adenomyotic tissues," American Journal of Obstetrics and Gynecology, vol. 169, no. 3, pp. 734-738, 1993.

[21] G. Liu, J. Li, and Y. Liu, "The effect of yitong xiao to the levels of TXB26-Keto-PGF1 $\alpha$ and $\beta$-EP in rat with endometriosis," Shandong Medication, vol. 50, no. 44, pp. 61-62, 2010. 


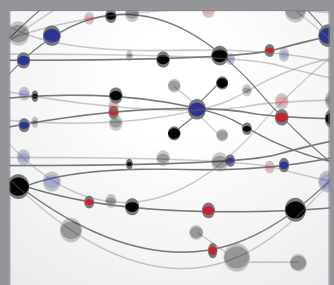

The Scientific World Journal
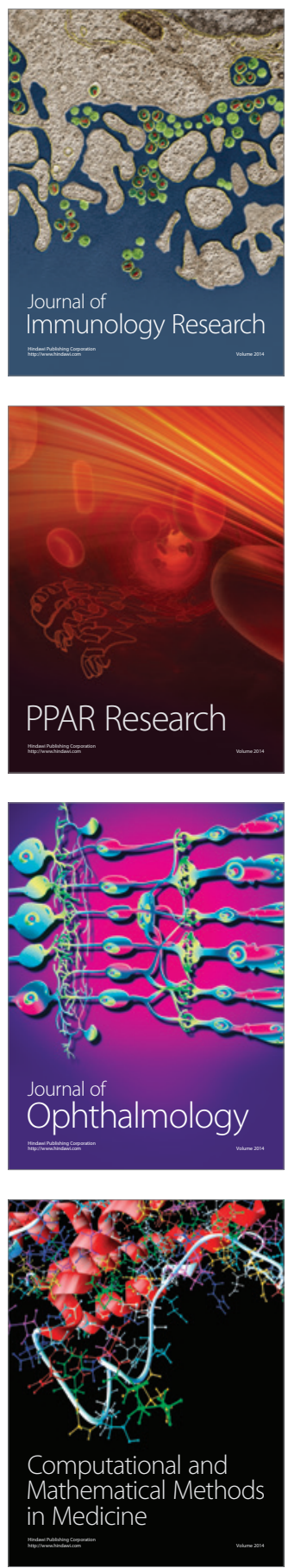

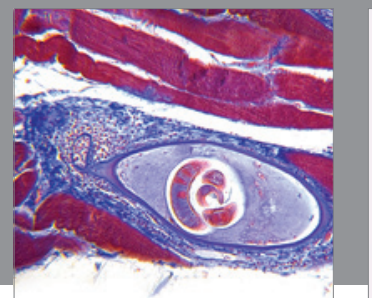

Gastroenterology

Research and Practice
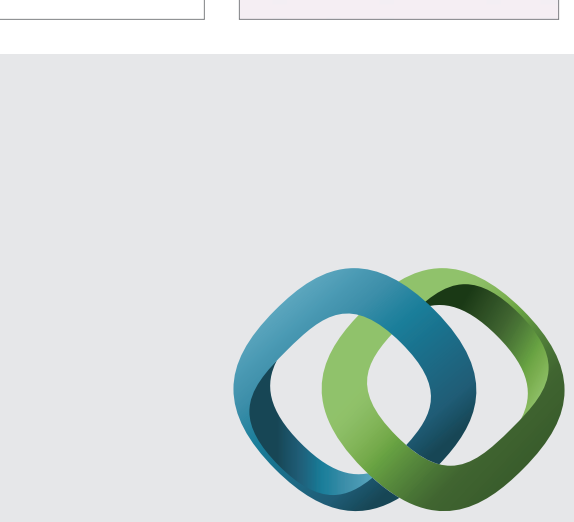

\section{Hindawi}

Submit your manuscripts at

http://www.hindawi.com
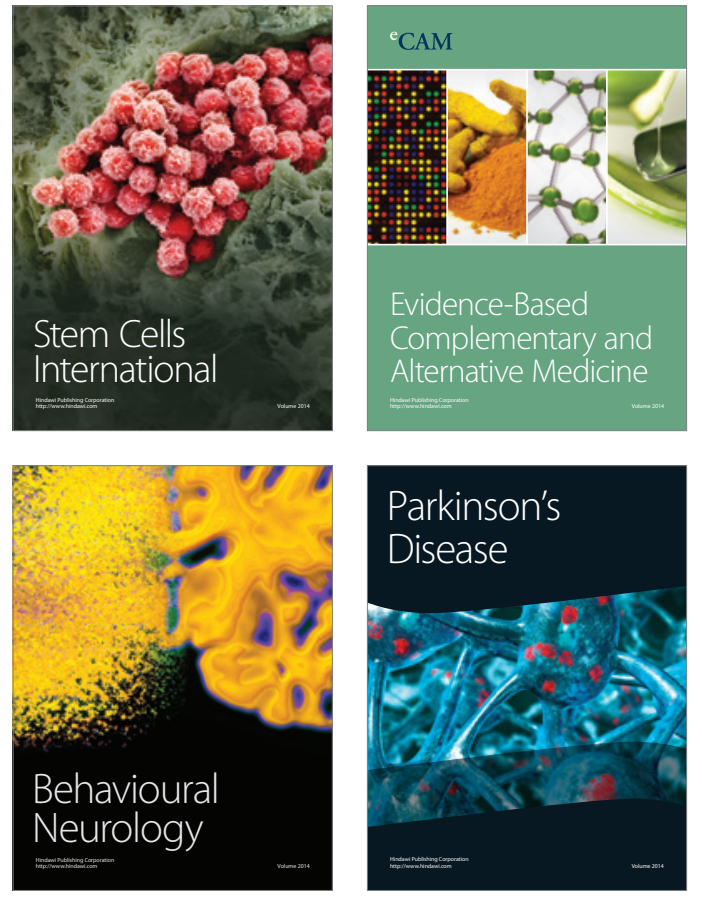
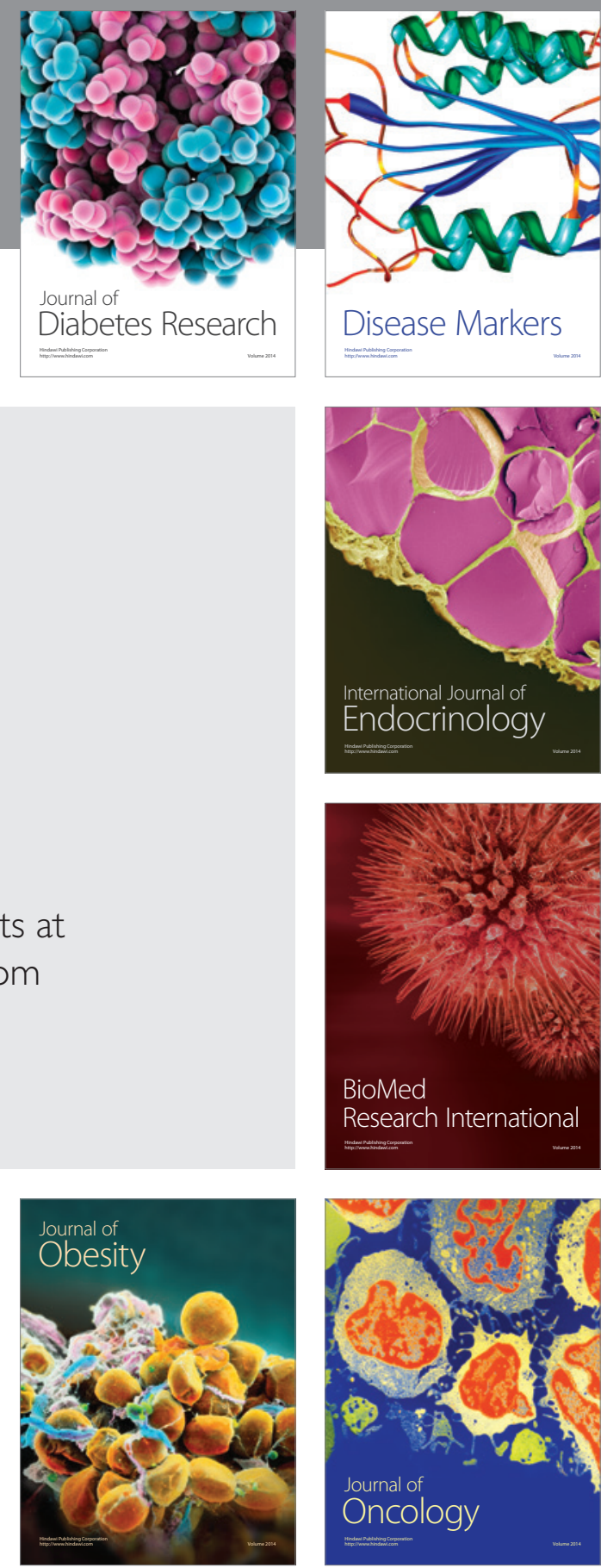

Disease Markers
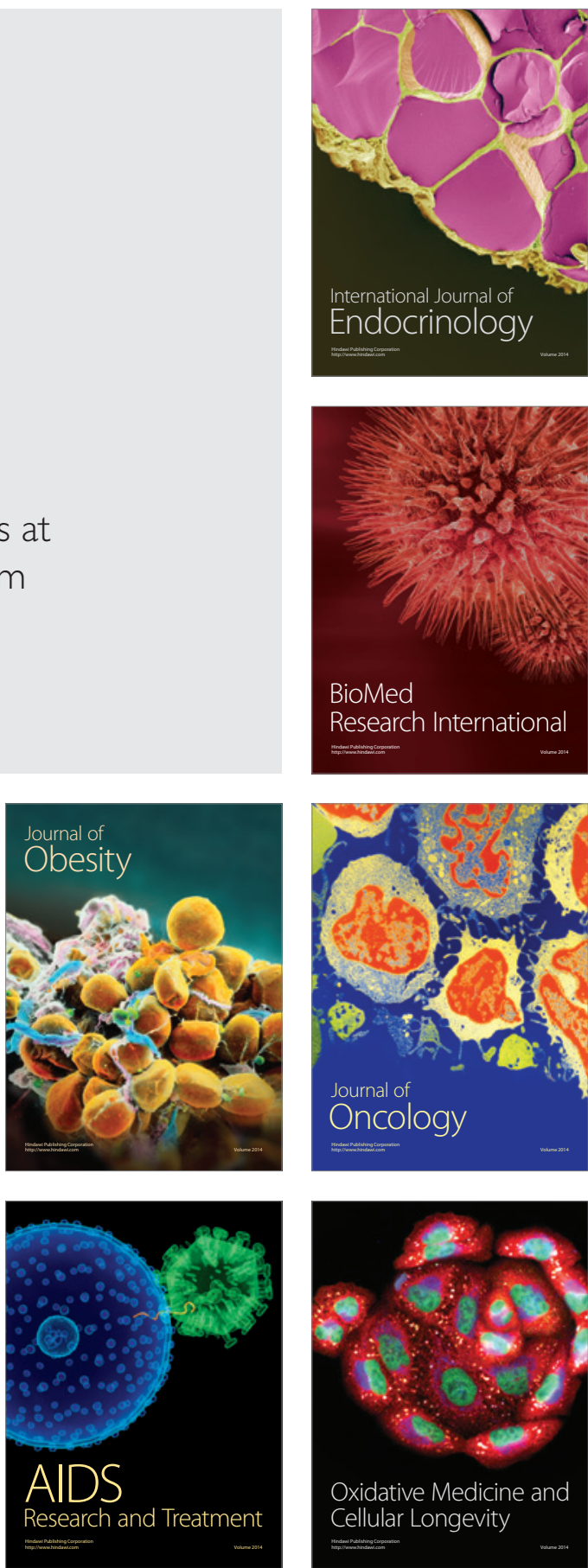